\title{
Colloidal Quantum Dot Light-Emitting Diodes Employing Phosphorescent Small Organic Molecules as Efficient Exciton Harvesters
}

\author{
Evren Mutlugun, ${ }^{\dagger, \ddagger, \S, \|}$ Burak Guzelturk, ${ }^{\dagger, \S, \|}$ Agus Putu Abiyasa, ${ }^{\S}, \|$ Yuan Gao, ${ }^{\S}$ Xiao Wei Sun, ${ }^{\S}$ \\ and Hilmi Volkan Demir ${ }^{*}, \dagger, \S$
}

\begin{abstract}
${ }^{\dagger}$ Department of Electrical and Electronics Engineering, Department of Physics, UNAM - Institute of Materials Science and Nanotechnology, Bilkent University, Ankara 06800, Turkey

${ }^{\ddagger}$ Electrical-Electronics Engineering, Abdullah Gul University, Kayseri, Turkey TR-38039

${ }^{\S}$ Luminous! Center of Excellence for Semiconductor Lighting and Displays, School of Electrical and Electronic Engineering, School of Physical and Materials Sciences, Nanyang Technological University, Singapore 639798, Singapore
\end{abstract}

Supporting Information

ABSTRACT: Nonradiative energy transfer (NRET) is an alternative excitation mechanism in colloidal quantum dot (QD) based electroluminescent devices (QLEDs). Here, we develop hybrid highly spectrally pure QLEDs that facilitate energy transfer pumping via NRET from a phosphorescent small organic molecule-codoped charge transport layer to the adjacent QDs. A partially codoped exciton funnelling electron transport layer is proposed and optimized for enhanced QLED performance while exhibiting very high color purity of $99 \%$. These energy transfer pumped hybrid QLEDs demonstrate a 6-fold enhancement factor in the external quantum efficiency over the conventional QLED structure, in which energy transfer pumping is intrinsically weak.

SECTION: Physical Processes in Nanomaterials and Nanostructures
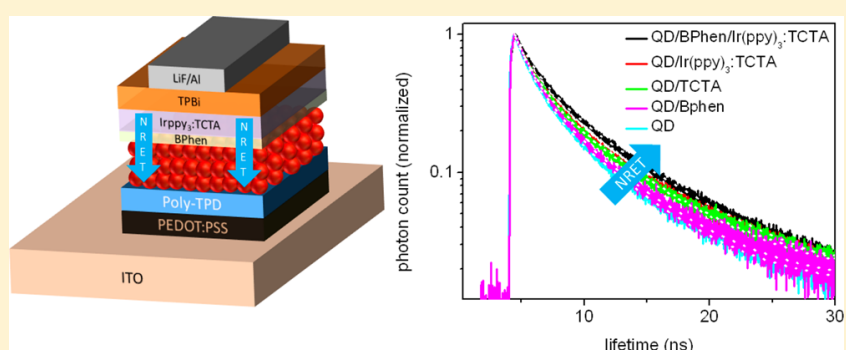

olloidal quantum dots (QDs) are appealing light-emitting materials owing to their favorable optical properties, which make them promising for electroluminescent lightemitting devices (QLEDs). ${ }^{1-3}$ Excitation of the QDs in these electroluminescent devices occurs via either direct charge injection and/or nonradiative energy transfer (NRET) from an adjacent charge transport layer. ${ }^{2}$ Energy transfer pumping was suspected to be an important mechanism for QLEDs, which employs organic hole and electron transport layers. ${ }^{4}$ Energy transfer pumping of the QDs can be facilitated through funnelling of excitons, which are initially formed in an adjacent transport layer, into the QDs via near field dipole-dipole coupling also known as Förster resonance energy transfer (FRET). Generally, these organic charge transport layers are comprised of fluorescent small organic molecules or conjugated polymers; therefore, only $1 / 4$ of the created excitons are singlet states due to spin statistics. ${ }^{5}$ Therefore, $3 / 4$ of the excitons ${ }^{6,7}$ that are formed in these fluorescent charge transport layers will not be transferred into the QDs via NRET since they have zero oscillatory strength. On the other hand, phosphorescent materials are known to have $100 \%$ internal quantum efficiency owing to the enhanced spin-orbit coupling and strong intersystem crossing. ${ }^{8,9}$ Hence, utilization of phosphorescent small organic molecules in the adjacent charge transport layer is expected to boost the energy transfer pumping of the QDs in QLEDs.

Previously, $\mathrm{NRET}^{10}$ and charge transfer ${ }^{11}$ from a phosphorescent small organic molecule to a QD was shown via timeresolved fluorescence spectroscopy. Phosphorescence lifetime was shown to be shortened due to NRET, while acceptor QD fluorescence lifetime is increased. ${ }^{12}$ Later, several reports demonstrated hybrid LEDs incorporating both the QDs and the phosphorescent small organic molecules for white light generation. ${ }^{13,14}$ However, these previous reports did not consider, nor engineer, the potential energy transfer pumping scheme. Only Zhang et al. reported an enhancement of the external quantum efficiency (EQE) up to 3-fold via employing triplet harvesting phosphorescent molecules, but the color (spectral) purity of QLEDs could not be preserved at high current densities due to the expansion of the exciton recombination zone resulting in incomplete energy transfer from the phosphorescent molecules into the QDs. ${ }^{15}$ In this work, we develop hybrid highly spectrally pure QLEDs, in which the QDs are energy transfer pumped via NRET from the

Received: June 20, 2014

Accepted: July 29, 2014

Published: July 29, 2014 


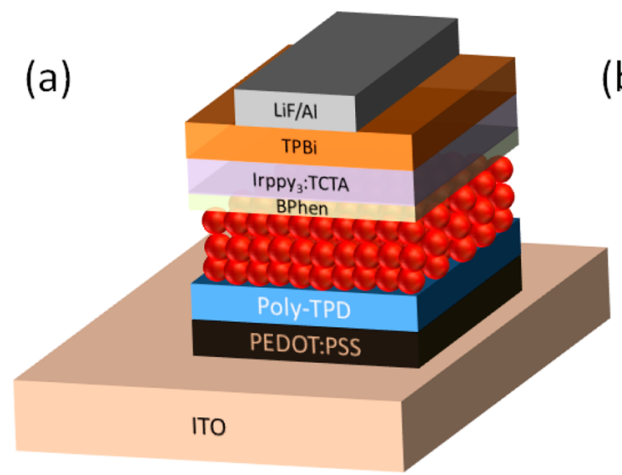

(b)

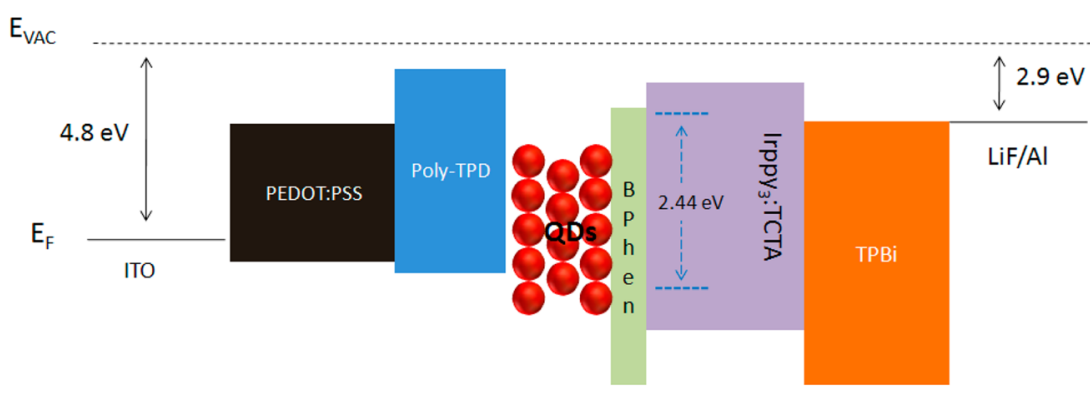

Figure 1. (a) 3D schematic of the QLED designed to achieve efficient energy transfer pumping. (b) Energy band alignment of the device in the structure of ITO/PEDOT:PSS/poly-TPD/QD/BPhen/Ir(ppy) ${ }_{3}:$ TCTA/TPBI/LiF/Al.

(a)

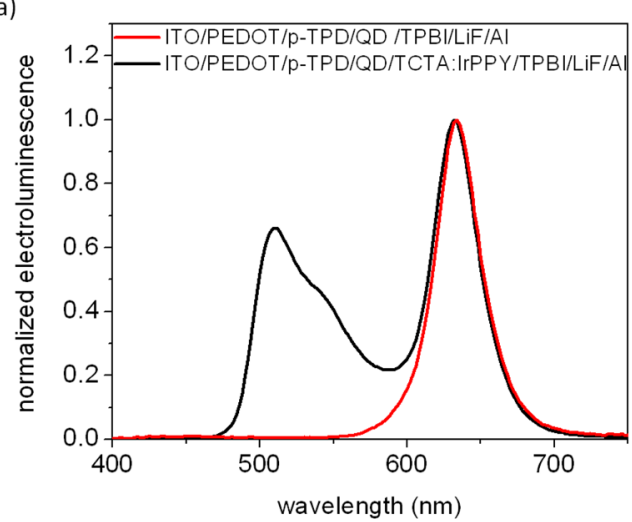

(b)

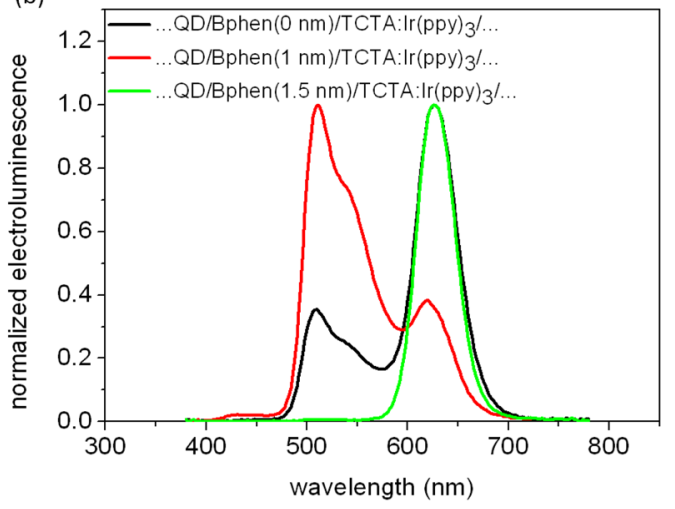

Figure 2. (a) Normalized electroluminescence spectra of the ITO/PEDOT:PSS/poly-TPD/QD/TPBI/LiF/Al, ITO/PEDOT:PSS/poly-TPD/QD/ TCTA:Ir(ppy) ${ }_{3} / \mathrm{TPBI} / \mathrm{LiF} / \mathrm{Al}$. (b) Normalized electroluminescence spectra of ITO/PEDOT:PSS/poly-TPD/QD/BPhen/TCTA:Ir(ppy) 3 /TPBI/ $\mathrm{LiF} / \mathrm{Al}$ with varying the BPhen thickness.

phosphorescent small organic molecule codoped electron transport layer. Excitonically engineered hybrid QLEDs exhibit substantially enhanced EQEs (up to $2.11 \%$ ) with more than 6fold enhancement over the conventional QLEDs that do not possess efficient exciton transferring phosphorescent codoped adjacent charge transport layers. These engineered hybrid QLEDs can preserve the color purity, which is as high as $99 \%$. Here, we denote the color purity as the fraction of photons emitted by the QDs to the total emitted photons from the overall hybrid QLED. The color purity of the electroluminescence of the hybrid QLEDs is successfully achieved via exciton formation zone management by introducing a hole blocking layer in between the QDs and the phosphorescent codoped electron transport layer. To optimize the energy transfer pumping to the QDs, location of the partial codoping of the phosphorescent molecules in the electron transport layer is fine-tuned. Additionally, time-resolved fluorescence spectroscopy is utilized to experimentally verify the enhanced exciton feeding into the QDs.

In this report, as opposed to the previous efforts on optimizing direct charge injection in QLEDs, our aim is to achieve high color purity QLEDs that are engineered for the highly efficient energy transfer pumping while effectively harvesting the singlet and triplet state excitons in the phosphorescent codoped electron transport layer. Red-emitting core/shell CdSe/ZnS QDs are synthesized ${ }^{16}$ and used as an emissive layer for the QLEDs. These QDs are highly monodisperse having less than $30 \mathrm{~nm}$ full-width at halfmaximum and exhibit $>20 \%$ quantum efficiency in their solid films. As the triplet state harvester and efficient energy transfer pump, a phosphorescent molecule-codoped electron transport layer of $2,2^{\prime}, 2^{\prime \prime}$-tris $(N$-carbazolyl)-triphenylamine:iridium, tris(2-phenylpyidine) (TCTA:Ir $(\text { ppy })_{3}$ ) is employed. The proposed 3D device architecture of the hybrid QLEDs is illustrated in Figure 1a. Figure $1 \mathrm{~b}$ demonstrates the energy band alignments of the hybrid QLEDs.

The devices are fabricated using both solution and vacuum processing techniques (see Experimental Section for the details). Poly(3,4-ethylenedioxythiophene)-poly(styrenesulfonate) (PEDOT:PSS), poly[N,N'-bis(4-butylphenyl)-N,N'-bis(phenyl)benzidine] (poly-TPD), and the QDs are spin coated. Bathophenanthroline (BPhen), TCTA, $\operatorname{Ir}(\text { ppy })_{3}$, 1,3,5-tris( $N$-phenylbenzimiazole-2-yl)benzene (TPBI), and LiF are deposited using thermal sublimation at very high vacuum $\left(<10^{-6}\right.$ Torr $)$. The thickness of the deposited film is monitored using a calibrated quartz crystal microbalance (QCM) thickness gauge having sensitivity of $\pm 0.03 \mathrm{~nm}$. In the conventional device structure of indium tin oxide (ITO)/PEDOT:PSS/poly$\mathrm{TPD} / \mathrm{QD} / \mathrm{TPBI} / \mathrm{LiF} / \mathrm{Al}$, the emission is purely from the $\mathrm{QD}$ layer, as shown in Figure 2a. However, the maximum EQE level is quite low (i.e., $0.58 \%$ ), possibly due to poor charge injection balance and poor energy transfer pumping, which the latter could take place either from poly-TPD or TPBI into the QDs. Starting with the conventional structure, we first introduce the phosphorescent emitter-codoped $\operatorname{Ir}(\text { ppy })_{3}$ :TCTA layer (10\% doping concentration) (ITO/PEDOT:PSS/poly-TPD/QD/ TCTA:Ir(ppy) 3 / TPBI/LiF/Al) as the electron transport layer. However, we observe insuppressible emission from the 
phosphorescent codoping in the electroluminescence spectra, as shown in Figure 2a. This necessitates engineering of the exciton recombination zone to achieve efficient exciton confinement to the QD layer for superior color purity. To this end, we introduce a BPhen hole-blocking layer to keep the electroluminescence spectrally pure while optimizing the energy transfer pumping of the QDs via minimizing the leakage of the holes from the $\mathrm{QD}$ layer into the phosphorescent-codoped layer. As we introduce the BPhen layer in between the $\mathrm{QD}$ layer and the codoped layer (ITO/ PEDOT:PSS/poly-TPD/QD/BPhen/TCTA:Ir(ppy) 3 /TPBI/ $\mathrm{LiF} / \mathrm{Al}$ ), we observe a significant suppression of the emission from the phosphorescent molecules. As we increase the thickness of BPhen from 0.5 to $1.5 \mathrm{~nm}$, the electroluminescence spectra of the hybrid QLEDs are shown in Figure $2 \mathrm{~b}$. Therefore, we achieve as high as $99 \%$ color purity (fraction of the photons emitted by QDs to the total number of photons emitted by the hybrid QLED) with the optimized $1.5 \mathrm{~nm}$ thick BPhen device (Figure $2 b$ green curve), whereas the fraction of the $\mathrm{QD}$ emission in the electroluminescence was just $76 \%$ without employing BPhen hole blocking layer (Figure $2 \mathrm{~b}$ black curve). This introduced very thin BPhen layer slightly increases the turn-on voltage of the hybrid QLEDs due to the impeded hole injection and transport (see Figure S1, Supporting Information). This result implies the substantial suppression of the undesired photons (i.e., emission of the phosphorescent small organic molecules) from $24 \%$ to $1 \%$. It is worth noting in here that the thickness of BPhen plays a critical role in determining the overall device efficiency. While a thicker BPhen layer provides a better color purity, exciton transfer from the phosphorescent molecules to the QDs is expected to be suppressed. Varying the BPhen layer thickness from 0 to $2 \mathrm{~nm}$, the optimized device performance is achieved for a BPhen thickness of $1.5 \mathrm{~nm}$. At this optimum condition, the hybrid QLEDs both maintain high EQEs without sacrificing the excellent color purity.

To further engineer the energy transfer pumping in these hybrid QLEDs, we study a partially codoped $\operatorname{Ir}(\mathrm{ppy})_{3}$ :TCTA electron transport layer instead of a completely codoped layer while keeping all other layers at the optimal thicknesses. Here, we codope $\operatorname{Ir}(\mathrm{ppy})_{3}$ into TCTA with a thickness of $2.5 \mathrm{~nm}$, and the rest of the TCTA is kept undoped $(7.5 \mathrm{~nm})$. Figure 3 exhibits the representative schematic of these partially codoped electron transport layers. Then, we change the position (i.e., 1 to 4 as in Figure 3) of this codoped part inside the TCTA layer. This enables us to probe the effects of the energy transfer pumping on the electroluminescence and EQE of the QLEDs. As the codoped portion is closer to the QDs, it is expected to achieve enhanced energy transfer pumping. Figure 4a shows the change in the resulting non-normalized electroluminescence spectra of the QLEDs at the same injection current density as we vary the $2.5 \mathrm{~nm}$ thick codoping layer's position in $2.5 \mathrm{~nm}$ intervals within the $10 \mathrm{~nm}$ film thickness of TCTA. As the codoping is placed closest to the QDs (position 1), we achieve $99 \%$ color purity (emission ratio of the QDs to $\operatorname{Ir}(\mathrm{ppy})_{3}$ is $\sim 100$ ) with the highest overall emission intensity as shown by Figure 4a. Moreover, as we move the codoping away (positions 2 to 4) from the QDs, the color purity becomes inferior (see Figure $4 \mathrm{a}$ inset), and we observe that the color purity quantitatively decreases from $99 \%$ to $92 \%$. This is due to the fact that energy transfer pumping from the phosphorescent codoping to the QDs decreases, and the excitons that are generated in the codoping start to recombine radiatively instead

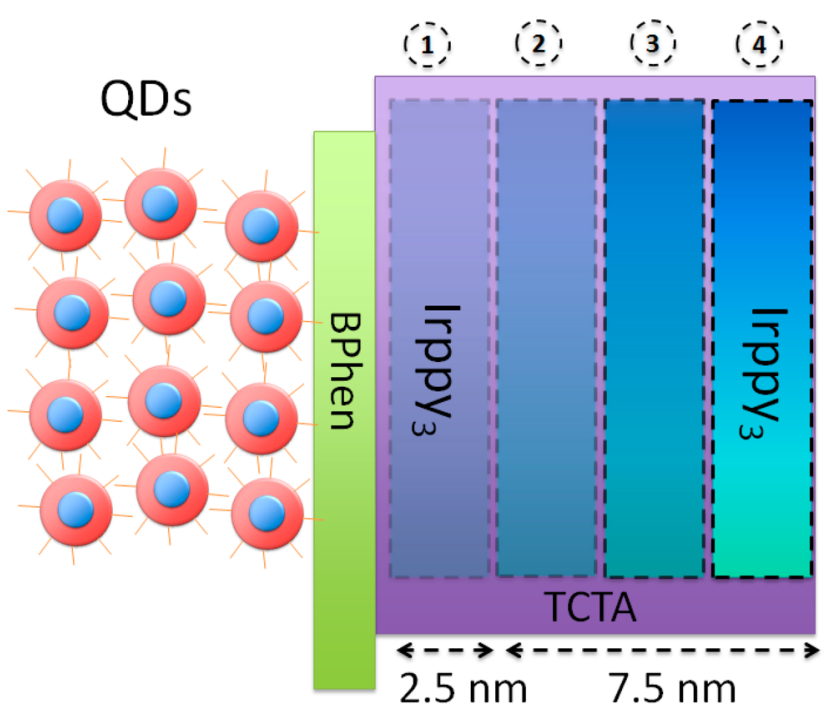

Figure 3. Schematic of the partially phosphorescent codoped electron transport layer of TCTA. The partial codoping is positioned in four different zones within the TCTA as indicated by positions 1 to 4 above the scheme, where position 1 is the closest to the QDs. NRET is expected to be the strongest for the codoping located at position 1 .

of being funneled into the QDs. Figure $4 \mathrm{~b}$ shows the EQE of four different hybrid QLEDs at the same current density (55 $\mathrm{mA} / \mathrm{cm}^{2}$ ) having varying codoping position. The closest codoping leads to the best EQE levels. The EQEs progressively decrease as the codoping is moved from position 1 to 4 .

For the well optimized case of the codoping layer being closest to the QDs (position of the codoping is 1) (ITO/ PEDOT:PSS/poly-TPD/QD/BPhen (1.5 nm)/TCTA:Ir$\left.(\text { ppy })_{3}(2.5 \mathrm{~nm}) / \mathrm{TCTA}(7.5 \mathrm{~nm}) / \mathrm{TPBI} / \mathrm{LiF} / \mathrm{Al}\right)$, the peak the EQE of the device reaches $2.11 \%$, which is 3.6 -fold larger than peak EQE of the conventional QLED, in which peak EQEs can only reach $0.58 \%$ as shown by Figure $4 \mathrm{c}$. The maximum enhancement factor achieved in EQE is over 6-fold at the current density of $11 \mathrm{~mA} / \mathrm{cm}^{2}$. Figure $4 \mathrm{~d}$ shows the electroluminescence spectra of the QLED at increasing current levels (device area is $9 \mathrm{~mm}^{2}$ ) that is optimized for energy transfer pumping and high color purity. Furthermore, the optimized device results in peak brightness level as high as 1574 $\mathrm{cd} / \mathrm{m}^{2}$, as shown in Figure S2. Table 1 summarizes various combinations of the devices under consideration along with their EQE levels.

To support the observed trends in the device performance of the hybrid QLEDs owing to the efficient energy transfer pumping by the phosphorescent codoped layer, we further investigate these hybrid thin films via time-resolved fluorescence spectroscopy. Previously, fluorescence of the QDs was shown to be enhanced owing to NRET. ${ }^{17-19}$ Here, NRET is employed to increase the electroluminescence performance of the electrically driven QDs. Figure 5 shows the fluorescence decay curves measured at the QD peak emission to investigate the fluorescence lifetime variation of the QDs due to NRET from the additional layers of that are employed in the QLEDs including BPhen, TCTA, $\operatorname{Ir}(\text { ppy })_{3}:$ TCTA, and BPhen/Ir(ppy) $)_{3}:$ TCTA. The collected fluorescence decays are analyzed using biexponential decay function. It is expected to have increased fluorescence lifetime of the acceptor QDs owing to the exciton feeding via NRET. ${ }^{12,19}$ The fluorescence lifetime of the QDs increases from $2.79 \mathrm{~ns}$ (amplitude averaged lifetime in 

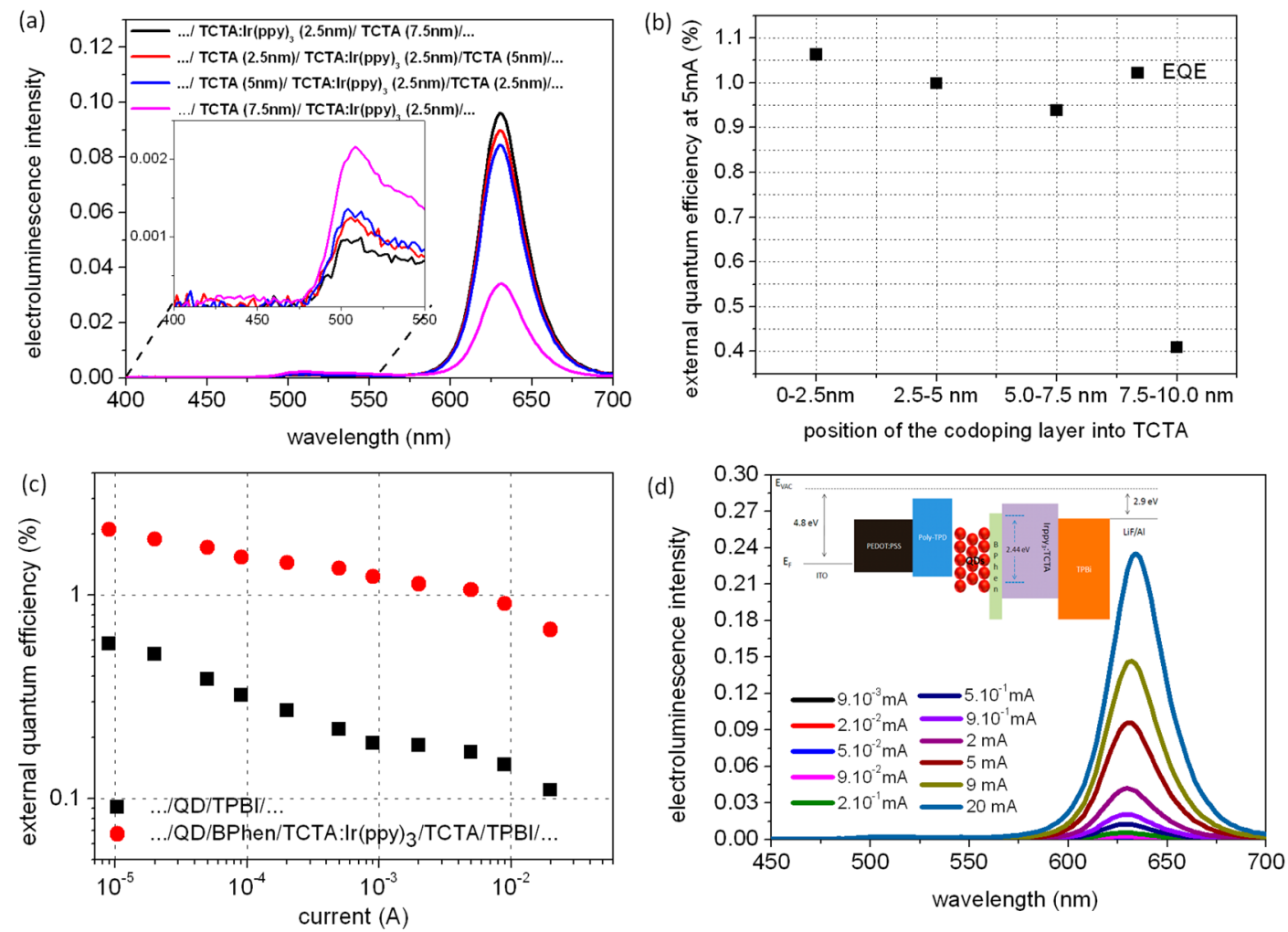

Figure 4. (a) Non-normalized electroluminescence spectra of the ITO/PEDOT:PSS/poly-TPD/QD/BPhen/TCTA:Ir(ppy) ${ }_{3} / \mathrm{TPBI} / \mathrm{LiF} / \mathrm{Al}$ device with varying the position of the codoped TCTA:Ir(ppy) layer within the TCTA electron transport layer. (b) EQEs of the devices at $5 \mathrm{~mA}$ injection current as a function of the position of the codoping layer into TCTA. (c) EQE vs current density of the devices: energy transfer pumping and color purity optimized ITO/PEDOT:PSS/poly-TPD/QD/BPhen(1.5 nm)/TCTA:Ir(ppy) ${ }_{3}(2.5 \mathrm{~nm}) / \mathrm{TCTA}(7.5 \mathrm{~nm}) / \mathrm{TPBI} / \mathrm{LiF} / \mathrm{Al}$ and conventional ITO/PEDOT:PSS/poly-TPD/QD/TPBI/LiF/Al. (d) Electroluminescence spectra of the outperforming ITO/PEDOT:PSS/poly-TPD/QD/ BPhen/TCTA:Ir(ppy $)_{3} / \mathrm{TPBI} / \mathrm{LiF} / \mathrm{Al}$ device $\left(\operatorname{Ir}(\mathrm{ppy})_{3}\right.$ doping in the first $2.5 \mathrm{~nm}$ of the TCTA layer).

Table 1. Maximum EQE Values of the Devices under Consideration

\begin{tabular}{|c|c|}
\hline device & $\underset{(\%)}{\max .} \operatorname{EQE}$ \\
\hline ITO/PEDOT:PSS/poly-TPD/TCTA:Ir(ppy) 3 /TPBI/LiF/Al & 14.66 \\
\hline $\begin{array}{l}\text { ITO/PEDOT:PSS/poly-TPD/BPhen/TCTA:Ir(ppy) }{ }_{3} / \mathrm{TPBI} / \\
\mathrm{LiF} / \mathrm{Al}\end{array}$ & 8.28 \\
\hline ITO/PEDOT:PSS/poly-TPD/QD/TPBI/LiF/Al & 0.58 \\
\hline ITO/PEDOT:PSS/poly-TPD/QD/TCTA/TPBI/LiF/Al & 0.77 \\
\hline ITO/PEDOT:PSS/poly-TPD/QD/BPhen/TPBI/LiF/Al & 0.79 \\
\hline $\begin{array}{l}\text { ITO/PEDOT:PSS/poly-TPD/QD/BPhen/TCTA/TPBI/LiF/ } \\
\mathrm{Al}\end{array}$ & 1.81 \\
\hline $\begin{array}{l}\text { ITO/PEDOT:PSS/poly-TPD/QD/TCTA:Ir(ppy) } 3 \text { /TPBI/ } \\
\text { LiF/Al }\end{array}$ & 3.22 \\
\hline $\begin{array}{l}\text { ITO/PEDOT:PSS/poly-TPD/QD/BPhen/TCTA:Ir(ppy) } 3 \text { / } \\
\text { TPBI/LiF/Al }\end{array}$ & 2.11 \\
\hline
\end{tabular}

bare QD film) when incorporated with exciton donating phosphorescent codoped charge transport layers. Table 2 summarizes the fluorescence lifetime of the QDs for the different donor cases. It has been shown that the longest fluorescence lifetime (3.87 ns) is achieved for the QD/BPhen/ $\operatorname{Ir}(\mathrm{ppy})_{3}$ :TCTA sample, which also resulted in the best QLED performance, with a $38.7 \%$ enhancement of the fluorescence lifetime of the QDs as compared to the lifetime of the bare QDs. The next longest lifetime is observed for the case of the $\mathrm{QD} / \mathrm{Ir}(\text { ppy })_{3}$ :TCTA sample (3.44 ns). Thus, the presence of the BPhen hole-blocking interlayer is observed to enhance the NRET to the QDs. We attribute this observation to the suppression of the interface trapping and nonradiative

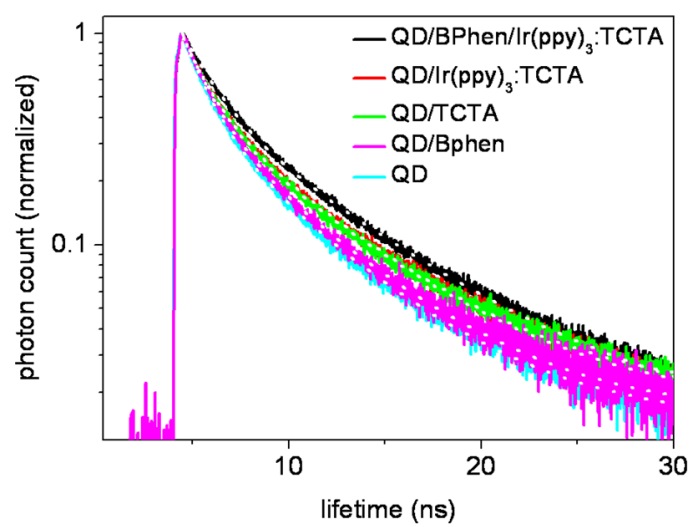

Figure 5. Time resolved fluorescence spectra of the $\mathrm{QD}$ layer in the bare and in various architecture samples (QD/BPhen, QD/TCTA, $\mathrm{QD} / \operatorname{Ir}(\mathrm{ppy})_{3}: \mathrm{TCTA}$, and QD/BPhen/Ir(ppy $\left.)_{3}: \mathrm{TCTA}\right)$ along with the biexponential fits given with white dots.

Table 2. Amplitude Weighted Fluorescence Lifetimes of the QD, QD/BPhen, QD/TCTA,QD/IrIr(ppy) ${ }_{3}$ :TCTA, and QD/BPhen/Ir(ppy) $)_{3}:$ TCTA Samples

\begin{tabular}{lc}
\multicolumn{1}{c}{ sample name } & amplitude weighted lifetime (ns) \\
QD & 2.79 \\
QD/BPhen & 2.94 \\
QD/TCTA & 3.26 \\
QD $/ \operatorname{Ir}(\text { ppy })_{3}:$ TCTA & 3.44 \\
QD/BPhen $/ \operatorname{Ir}(\text { ppy })_{3}:$ TCTA & 3.87
\end{tabular}


dissociation of the triplet excitons at the interface. ${ }^{20}$ When BPhen is employed as an interlayer at the interface, trapping and nonradiative dissociation of the triplet excitons may be possibly suppressed.

In conclusion, we demonstrated efficient energy transfer pumping of the QDs by NRET from the phosphorescent codoped electron transport layer. This energy transfer pumping via exciton harvesting boosts up the efficiency of the QLEDs while maintaining the color purity (as high as 99\%) of the devices via controlling the exciton formation zone in the excitonically engineered devices. The results reveal up to a 6fold enhancement of the EQEs when the phosphorescent layer is introduced in the engineered architecture as compared to the conventional QLEDs. The utilization of the exciton harvesting in such architectures will pave the way for the superior performance of electroluminescent devices.

\section{EXPERIMENTAL SECTION}

Device Fabrication. For all devices under consideration, patterned ITO glass (sheet resistance of $25 \Omega / \mathrm{sq}$ ) is first cleaned using deionized (DI) water, acetone, and isopropanol followed by the oxygen plasma treatment prior to PEDOT:PSS spin coating. The device fabrication steps are all performed inside a $\mathrm{N}_{2}$ filled glovebox environment without breaking the vacuum during the deposition of subsequent organic molecules. Spin coating of PEDOT:PSS and poly-TPD $(15 \mathrm{mg} / \mathrm{mL}$ in chlorobenzene) is performed using $4 \mathrm{k} \mathrm{rpm}$ for $1 \mathrm{~min}$ followed by baking at $100{ }^{\circ} \mathrm{C}$. Quantum dots in toluene $(10 \mathrm{mg} / \mathrm{mL})$ is spin coated using the same recipe for PEDOT:PSS and polyTPD with baking at $80{ }^{\circ} \mathrm{C}$ for $30 \mathrm{~min}$. After the solution process steps are completed, samples are loaded into organic evaporation chamber to deposit layers under consideration. In general approach, unless otherwise stated, the thicknesses of the organic and metal layers have been chosen as follows: TCTA:Ir(ppy $)_{3}$ is deposited using $10 \%$ codoping of $\operatorname{Ir}($ ppy $) 3$ into TCTA with a total film thickness of $2.5 \mathrm{~nm}$; undoped TCTA as $7.5 \mathrm{~nm}$, TPBI as $35 \mathrm{~nm}$, BPhen varied as 1, 1.5, and 2 $\mathrm{nm}$; lithium floride $(\mathrm{LiF})$ as $1 \mathrm{~nm}$, and Aluminum (Al) as 150 $\mathrm{nm}$.

Device Measurement. The emission spectra of the devices fabricated were measured using Photo Research Spectra Scan PR705 spectra photometer and Konica-Minolta LS-100 luminance-meter. The time resolved fluorescence spectroscopy measurements were carried out using Becker-Hickl system. The devices were characterized under ambient conditions without any encapsulation.

\section{ASSOCIATED CONTENT}

\section{S Supporting Information}

Additional information on the current versus voltage curve of the device with or without BPhen, and the luminance versus current curve of the optimized device. This material is available free of charge via the Internet at http://pubs.acs.org.

\section{AUTHOR INFORMATION}

\section{Corresponding Author}

*E-mail: volkan@bilkent.edu.tr or hvdemir@ntu.edu.sg.

\section{Author Contributions}

"E.M., B.G., and A.P.A. contributed equally to this work.

\section{Notes}

The authors declare no competing financial interest.

\section{ACKNOWLEDGMENTS}

The authors would like to acknowledge the financial support from the Singapore National Research Foundation under the programs of NRF-RF-2009-09 and NRF-CRP-6-2010-02, the Science and Engineering Research Council, Agency for Science, Technology and Research (A*STAR) of Singapore (Project Nos. 61006037 and 61076015), EU-FP7 Nanophotonics4Energy NoE, TUBITAK EEEAG 109E002, 109E004, 110E010, 110E217, ESF-EURYI, and TUBA-GEBIP. E.M. acknowledges BAGEP award

\section{REFERENCES}

(1) Coe, S.; Woo, W.-K.; Bawendi, M.; Bulović, V. Electroluminescence from Single Monolayers of Nanocrystals in Molecular Organic Devices. Nature 2002, 420, 800-803.

(2) Shirasaki, Y.; Supran, G. J.; Bawendi, M. G.; Bulović, V. Emergence of Colloidal Quantum-Dot Light-Emitting Technologies. Nat. Photonics 2012, 7, 13-23.

(3) Dang, C.; Lee, J.; Breen, C.; Steckel, J. S.; Coe-Sullivan, S.; Nurmikko, A. Red, Green and Blue Lasing Enabled by Single-Exciton Gain in Colloidal Quantum Dot Films. Nat. Nanotechnol. 2012, 7, 335-339.

(4) Anikeeva, P.; Madigan, C.; Halpert, J.; Bawendi, M.; Bulović, V. Electronic and Excitonic Processes in Light-Emitting Devices Based on Organic Materials and Colloidal Quantum Dots. Phys. Rev. B 2008, 78, 085434.

(5) Baldo, M. A.; O’Brien, D. F.; Forrest, S. R. Excitonic SingletTriplet Ratio in a Semiconducting Organic Thin Film. Phys. Rev. B 1999, 60, 14422-14428.

(6) Köhler, A.; Bässler, H. Triplet States in Organic Semiconductors. Mater. Sci. Eng. R Reports 2009, 66, 71-109.

(7) Reineke, S.; Thomschke, M.; Lüssem, B.; Leo, K. White Organic Light-Emitting Diodes: Status and Perspective. Rev. Mod. Phys. 2013, $85,1245-1293$

(8) Baldo, M. A.; O’Brien, D. F.; You, Y.; Shoustikov, A.; Sibley, S.; Thompson, M. E.; Forrest, S. R. Highly Efficient Phosphorescent Emission from Organic Electroluminescent Devices. Nature 1998, 395, $151-154$.

(9) Adachi, C.; Baldo, M. A.; Thompson, M. E.; Forrest, S. R. Nearly $100 \%$ Internal Phosphorescence Efficiency in an Organic LightEmitting Device. J. Appl. Phys. 2001, 90, 5048.

(10) Anikeeva, P. O.; Madigan, C. F.; Coe-Sullivan, S. A.; Steckel, J. S.; Bawendi, M. G.; Bulović, V. Photoluminescence of CdSe/ZnS Core/Shell Quantum Dots Enhanced by Energy Transfer from a Phosphorescent Donor. Chem. Phys. Lett. 2006, 424, 120-125.

(11) Wang, Y.; Li, S.; Kershaw, S. V.; Hetsch, F.; Tam, A. Y. Y.; Shan, G.; Susha, A. S.; Ko, C.-C.; Wing-Wah Yam, V.; Lo, K. K. W.; et al. Design of a Water-Soluble Hybrid Nanocomposite of CdTe Quantum Dots and an Iridium Complex for Photoinduced Charge Transfer. ChemPhysChem 2012, 13, 2589-2595.

(12) Kagan, C.; Murray, C.; Nirmal, M.; Bawendi, M. Electronic Energy Transfer in CdSe Quantum Dot Solids. Phys. Rev. Lett. 1996, $76,1517-1520$.

(13) Cheng, G.; Mazzeo, M.; Rizzo, A.; Li, Y.; Duan, Y.; Gigli, G. White Light-Emitting Devices Based on the Combined Emission from Red CdSe/ZnS Quantum Dots, Green Phosphorescent, and Blue Fluorescent Organic Molecules. Appl. Phys. Lett. 2009, 94, 243506.

(14) Cheng, G.; Lu, W.; Chen, Y.; Che, C.-M. Hybrid Light-Emitting Devices Based on Phosphorescent Platinum(II) Complex Sensitized CdSe/ZnS Quantum Dots. Opt. Lett. 2012, 37, 1109-1111.

(15) Zhang, Y. Q.; Cao, X. A. Electroluminescence of Green CdSe/ ZnS Quantum Dots Enhanced by Harvesting Excitons from Phosphorescent Molecules. Appl. Phys. Lett. 2010, 97, 253115.

(16) Bae, W. K.; Kwak, J.; Lim, J.; Lee, D.; Nam, M. K.; Char, K.; Lee, C.; Lee, S. Multicolored Light-Emitting Diodes Based on AllQuantum-Dot Multilayer Films Using Layer-by-Layer Assembly Method. Nano Lett. 2010, 10, 2368-2373. 
(17) Achermann, M.; Petruska, M. A.; Kos, S.; Smith, D. L.; Koleske, D. D.; Klimov, V. I. Energy-Transfer Pumping of Semiconductor Nanocrystals Using an Epitaxial Quantum Well. Nature 2004, 429, 642-646.

(18) Nizamoglu, S.; Guzelturk, B.; Jeon, D.-W.; Lee, I.-H.; Demir, H. V. Efficient Nonradiative Energy Transfer from InGaN/GaN Nanopillars to CdSe/ZnS Core/Shell Nanocrystals. Appl. Phys. Lett. 2011, 98, 163108 .

(19) Guzelturk, B.; Martinez, P. L. H.; Zhang, Q.; Xiong, Q.; Sun, H.; Sun, X. W.; Govorov, A. O.; Demir, H. V. Excitonics of Semiconductor Quantum Dots and Wires for Lighting and Displays. Laser Photon. Rev. 2014, 8, 73-93.

(20) Ribierre, J.; Ruseckas, A.; Knights, K.; Staton, S.; Cumpstey, N.; Burn, P.; Samuel, I. Triplet Exciton Diffusion and Phosphorescence Quenching in Iridium(III)-Centered Dendrimers. Phys. Rev. Lett. 2008, 100, 017402. 